Mathematisches Forschungsinstitut Oberwolfach

Report No. $31 / 2016$

DOI: $10.4171 /$ OWR/2016/31

\title{
Algebraic K-theory and Motivic Cohomology
}

\author{
Organised by \\ Thomas Geisser, Tokyo \\ Annette Huber-Klawitter, Freiburg \\ Uwe Jannsen, Regensburg \\ Marc Levine, Essen
}

26 June - 2 July 2016

\begin{abstract}
Algebraic $K$-theory and motivic cohomology have developed together over the last thirty years. Both of these theories rely on a mix of algebraic geometry and homotopy theory for their construction and development, and both have had particularly fruitful applications to problems of algebraic geometry, number theory and quadratic forms. The homotopy-theory aspect has been expanded significantly in recent years with the development of motivic homotopy theory and triangulated categories of motives, and $K$-theory has provided a guiding light for the development of non-homotopy invariant theories. 19 one-hour talks presented a wide range of latest results on many aspects of the theory and its applications.
\end{abstract}

Mathematics Subject Classification (2010): 14Cxx, 14Fxx, 19Axx, 19Exx, 19Fxx.

\section{Introduction by the Organisers}

Algebraic $K$-theory and motivic cohomology have developed together over the last thirty years. Both of these theories rely on a mix of algebraic geometry and homotopy theory for their construction and development, and both have had particularly fruitful applications to problems of algebraic geometry, number theory and quadratic forms. The homotopy-theory aspect has been expanded significantly in recent years with the development of motivic homotopy theory and triangulated categories of motives, and $K$-theory has provided a guiding light for the development of non-homotopy invariant theories.

The workshop program presented a varied series of lectures on the latest developments in the field. The 53 participants came mostly from Europe, but there were large contingents from the USA and Japan, as well as additional participants 
from Argentina, Russia and India. The participants ranged from leading experts in the field to younger researchers and also some graduate students. 19 one-hour talks presented a wide range of the latest results on the theory and its applications, reflecting a good mix of nationalities and age groups.

Here is a more detailed description of the talks.

Computations in $K$-theory and in the homology of linear groups. Hesselholt (with M. Larsen and A. Lindestrauss) constructed a reduced norm map for $p$-adic $K$-theory of a division ring over a local field with residue field of characteristic $p>0$, using the cyclotomic trace map. Morrow extended known results on mod $p^{n}$ - $K$-theory to the $p$-adic case and used this to prove continuity results, infinitesimal versions of weak Lefschetz for the Chow groups and a $p$-adic version of a conjecture of Kato-Saito. Wendt described his work giving a presentation of the homology of $\mathrm{GL}_{3}$ of a elliptic curve and its relation to the construction of an elliptic dilogarithm complex. Schlichting described a refinement of Suslin's results on the homology of $G L_{n}$ to the case of $S L_{n}$ and $E_{n}$.

Categorical constructions. Zakharevich described her approach to the study of $K_{0}(V a r)$ via a categorical scissors congruence construction. Yamazaki presented a framework (developed jointly with Kahn and Saito) for a triangulated category of "motives with modulus", which hopefully will give a good framework for studying non-homotopy invariant phenomena, such as wild ramification. Déglise showed how to construct a category of effective motives over a general base (joint with Bondarko), which admits a reasonable $t$-structure. Ivorra gave a description of the nearby cycles functor in terms of tubes in non-archimedean geometry. Panin presented aspects of his work with Garkusha on a new description of the motivic stable homotopy category in terms of "framed correspondences" and drew an analogy with Segal's machine for constructing infinite loop spaces.

Theories for topological rings. Tamme presented his work (with Kerz and Saito) describing the "generic fiber" of the comparison map from the $K$-theory of a smooth proper scheme over a complete DVR its continuous $K$-theory in terms of a $K$-theory of the associated rigid analytic space. Using the theory of bornological algebras, Cortiñas showed how to define a theory of rigid cohomology in the noncommutative setting, and gave a description of this in terms of cyclic homology (a joint work with Cuntz, Meyer and Tamme).

Motives and algebraic cycles. Kohrita extended the classical theory of cycles algebraically equivalent to zero and the notion of a universal regular homomorphism, for smooth projective varieties, to the case of smooth varieties and motivic cohomology. He showed the existence of the universal regular homomorphism to a semi-abelian variety for motivic cohomology in a certain range. Tabuada discussed non-commutative versions of Grothendieck's conjecture on the equality of numerical and homological equivalence, and Voevodsky's smash nilpotence conjecture. He showed that the non-commutative and commutative conjectures are equivalent, 
gave applications to quadric fibrations and stacks, and showed that under certain conditions, these conjectures are invariant under homological projective duality.

Ancona presented his work proving Grothendieck's conjecture on the positive definiteness of the intersection pairing in a number of new cases. Kahn showed how to interpret the Griffiths group of a smooth projective threefold $X$ (assuming a Chow-Künneth decomposition) as the group of morphisms in the category of motives modulo algebraic equivalence from the Lefschetz motive to the transcendental part of $h_{3}(X)$. Vial discussed the question: is the field of definition of the intermediate Jacobian (assuming this to be algebraic, or its algebraic part if it is not) of a smooth projective complex variety $X$ the same as the field of definition of $X$, and showed that this is the case for $J_{a l g}^{3}$. In addition, he showed (with Achter and Casalaina-Martin) that the associated Abel-Jacobi map is Galois equivariant.

Arithmetic. Schmidt (with Stix) considered a more general formulation of Grothendieck's section conjecture, involving the entire étale homotopy type rather than just the fundamental group, and obtained a number of "anabelian" statements for varieties that can be embedded as locally closed subschemes of a product of hyperbolic curves. Zhao gave an extension of duality in local class field theory to higher dimensional regular schemes in positive characteristic. Morin described a unified framework and series of conjectures (developed with Flach) for the zeta-values of arithmetic schemes.

Acknowledgement: The MFO and the workshop organizers would like to thank the National Science Foundation for supporting the participation of junior researchers in the workshop by the grant DMS-1049268, "US Junior Oberwolfach Fellows". Moreover, the MFO and the workshop organizers would like to thank the Simons Foundation for supporting Changlong Zhong in the "Simons Visiting Professors" program at the MFO. 



\section{Workshop: Algebraic K-theory and Motivic Cohomology}

\section{Table of Contents}

Lars Hesselholt (joint with Michael Larsen and Ayelet Lindenstrauss) $K$-theory of division algebras over local fields . . . . . . . . . . . 1759

Inna Zakharevich

The annihilator of the Lefschetz motive ..................... 1760

Tohru Kohrita

Algebraic part of motivic cohomology . . . . . . . . . . . . . . . . 1762

Matthew Morrow

Algebraic $K$-theory and motivic cohomology of formal schemes in

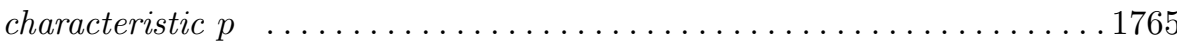

Gonçalo Tabuada

Remarks on Grothendieck's standard conjecture of type $D$ and on Voevodsky's nilpotence conjecture ....................... 1768

Giuseppe Ancona

On the standard conjecture of Hodge type for abelian varieties . . . . . . . 1771

Bruno Kahn

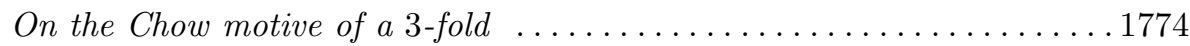

Alexander Schmidt (joint with Jakob Stix)

Anabelian geometry with étale homotopy types . . . . . . . . . . . . . . . . . 1775

Takao Yamazaki (joint with Bruno Kahn, Shuji Saito)

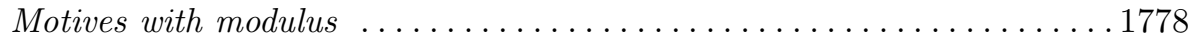

Baptiste Morin (joint with Matthias Flach)

Zeta-values of arithmetic schemes ..................... 1780

Guillermo Cortiñas (joint with Joachim Cuntz, Ralf Meyer, Georg Tamme)

Cyclic homology and rigid cohomology ................... 1783

Charles Vial (joint with Jeffrey Achter and Sebastian Casalaina-Martin)

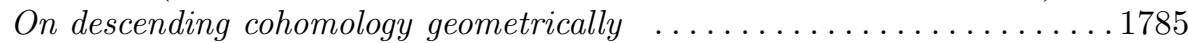

Yigeng Zhao

Étale duality for $p$-torsion sheaves on semistable schemes over $\mathbb{F}_{q}[[t]] \ldots 1787$

Georg Tamme (joint with Moritz Kerz, Shuji Saito)

K-theory for non-archimedean algebras and spaces ............. 1790

Frédéric Déglise (joint with Mikhail Bondarko)

Homotopy t-structure and effectivity 
Matthias Wendt

Homology of $\mathrm{GL}_{3}$ of elliptic curves

Florian Ivorra (joint with Joseph Ayoub and Julien Sebag)

Motives, nearby cycles and Milnor fibers ....................... 1797

Ivan Panin (joint with G.Garkusha)

A motivic version of Segal's theorem 1800

Marco Schlichting

Euler class groups and the homology of elementary groups 1801 